\title{
Effects of Combinational Procyanidins in Grape Seed Extract and Exercise on the Levels of Glucose and Blood Lipid Profile
}

Vega Vargas $\mathrm{F}^{1}$, Isabel Maria Guisado Requena ${ }^{2}$, Guillermo Arturo Canadas-De la Fuente ${ }^{3}$, Rafael Fernandez Castillo ${ }^{4}$, Fernandez Sanchez $\mathrm{E}^{4}$, Campos Jara $C^{5}$ and Rafael Guisado Barrilao ${ }^{6^{*}}$

${ }^{1}$ Program of PhD Physical activity and Health, Avda, de la llustración, Granada, Spain

${ }^{2}$ Program of PhD Nutrition and Sciences of the Food, University of Granada Avda, de la llustracion, Granada, Spain

${ }^{3}$ Department of Nursing, University of Granada Avda, de la Ilustracion, Granada, Spain

${ }^{4}$ Department of Nursing, University of Granada, Avda, de la llustracion, Granada, Spain

5 University Hospital of Virgen de las Nieves Granada, Spain

${ }^{6}$ Department of Nursing, Faculty of Sciences of Health, University of Granada, San Cecilio Clinical hospital, Avda, de la Ilustracion, Granada, Spain

"Corresponding authors: Rafael Guisado Barrilao, Faculty of Sciences of Health University of Granada, Department of Nursing, Avda. de Madrid, s/no 18012 Granada, Spain, Tel: 0034-609555284; E-mail: rguisado@ugr.es

Received date: August 03, 2017; Accepted date: August 16, 2017; Published date: August 20, 2017

Copyright: (C) 2017 Vega Vargas F, et al. This is an open-access article distributed under the terms of the Creative Commons Attribution License, which permits unrestricted use, distribution, and reproduction in any medium, provided the original author and source are credited.

\begin{abstract}
The oxygenation process is essential for life and cellular activity, and therefore for physical exercise. During physical exercise an oxidative stress situation is produced, which can result in cellular oxidation and alterations in the immune system, causing cells to age prematurely. To prevent this, it is recommended to use high concentrations of antioxidants to minimize this process.

The phenolic compounds of the flavonoid family, among which are the procyanidins, have shown neuroprotective effects, and cardioprotective effects. The cardiovascular protection and antiatherogenic effects of procyanidins have been attributed to the antioxidant properties of these phenolic compounds derived from its chemical structure. Different studies have shown the cardioprotective effects of chronic ingestion of procyanidins on models of hyperglycemia and dyslipidemia.

This study aims to present the properties of these compounds and their protective effects on the cardiovascular system, setting markers of the primary action of procyanidins from grape seed extract (obtained by discarding the pulp and preserving the seeds) in plasma. A secondary aim of this study was to provide information on the molecular mechanisms by which these substances exert their cardioprotective effect and implement these into a recommended dietary regime. This would be used in appropriate doses and complement the protective and regulatory effect of exercise.
\end{abstract}

Keywords: Antioxidants; Cardioprotective effect; Dietetic supplements; Physical exercise; Procyanidins

Abbreviations: GSPE: Grape seed procyanidins extract; GL: Glucose; CT: Entire cholesterol; c-HDL: Cholesterol tied to lipoproteins of high density; c-LDL: Cholesterol tied to lipoproteins of low density; TG: Triglycerides; VO2max: Maximal oxygen uptake; m: Meter; s: Seconds; min: Minutes; mg: Milligrams; Kg: Kilograms; GC: Control group; GP: Procyanidins group; GH: High calorie diet group; GEF: Physical exercise group; GPEF: Procyanidins and Physical exercise group

\section{Introduction}

Cardiovascular diseases constitute one of the main causes of morbidity and mortality in the world. There exists a series of factors; like physical, emotional, environmental and chemical stress, typical of modern life, that contribute to an epidemic of cardiovascular disease on a global scale [1]. In addition, the consumption of fats coming from our diet, particularly saturated fats and cholesterol, is related to the presence of diverse cardiovascular diseases, arteriosclerosis for example, that constitutes one of the prime movers of illness of coronary arteries and of cerebrovascular accidents [2].

The regular consumption of procyanidins in the flavonoid diet has been associated with a reduction in the mortality and in the risk of enduring cardiovascular illnesses [3]. Procyanidins have also been described as antioxidant substances with cardioprotective and hypolipidemic effects [4]. These compounds have the aptitude to modulate the activity of different enzymes and to interfere, consequently, in mechanisms of signalling and in different cellular processes which can result from its physicochemical characteristics that allow them to take part in different cellular metabolic oxide reduction reactions [5].

Furthermore, at present there is no doubt that moderate physical exercise, practised on a regular basis, since it has been demonstrated in numerous studies, provides a series of benefits for health and for the cardiovascular system, among these is a lipid lowering effect [6-8]. This constitutes an effective non-pharmacological measure in the treatment and in primary and secondary cardiovascular disease prevention [9], 
Citation: Vega Vargas F, Guisado Requena IM, Canadas-De la Fuente GA, Castillo RF, Fernandez Sanchez E, et al. (2017) Effects of Combinational Procyanidins in Grape Seed Extract and Exercise on the Levels of Glucose and Blood Lipid Profile. J Diabetes Metab 8: 754. doi:10.4172/2155-6156.1000754

Page 2 of 5

supposing a sedentary person's own inactivity to be an important factor in the development, maintenance and worsening of diverse chronic illnesses [10].

Following a review of the scientific literature on this matter, studies to date are scarce that have evaluated the effects of a treatment with procyanidins combined with a program of moderate physical exercise on blood biochemical parameters or on parameters related to the prevention of cardiovascular diseases. This has led to the realization of this study, bearing in mind the dimension of the problem that these illnesses pose for world health.

Therefore the present study aims to evaluate the effects of a treatment with grape seed procyanidin extract (GSPE), of a program of moderate physical exercise, and of the combination of both, on blood biochemical parameters (glucose-GL, entire cholesterol-CT, cholesterol tied to lipoproteins of low density-c-LDL, cholesterol tied to lipoproteins of high density-c-HDL and triglycerides-TG). We will also establish the therapeutic behaviours that provide information on its mechanism of action and its cardioprotective effects at plasma level.

\section{Material and methods}

\section{Experimentation animals}

A total of 25 adult male Wistar rats were used $(\mathrm{N}=25 ; 261,4 \pm 20.3$ grams) obtained from the animal department of the Centre of Scientific Instrumentation (CIC) of the University of Granada. All the experiments were conducted in accordance with the Guide for the Care and Use of Laboratory Animals (National Institutes of Health, NHI; publication No 85-23, examination in 1996) and it has been approved by the Institutional Committee for the Ethical Care of Animals of the University of Granada.

\section{Experimental design}

A 4-week randomized controlled trial was conducted. The sample was distributed randomly into 5 experimental groups of 5 animals in each one $(n=5)$ :

Control group (GC) submitted to normal conditions and diet (standard of commercial animal food for rats: Animal food A. 03 PANLAB S. L., Barcelona, Spain).

High calorie diet group (GH) fed with fatty sugary cream (Composition: Proteins $20 \%$, Carbohydrates 30\%, Fats 50\%).

Procyanidins group (GP) fed with fatty sugary cream and treated with GSPE (Procyanidin Source Naturals) in daily doses of $100 \mathrm{mg} / \mathrm{kg}$ of corporal weight a day [11,12] (dissolved in $1 \mathrm{ml}$ of distilled water), given orally by gavage in the same time slot $(10: 00 \mathrm{~h}-11: 00 \mathrm{~h})$.

Physical exercise group (GEF) fed with fatty sugary cream and submitted to a protocol of moderate physical exercise consisting of displacement to a treadmill (LE8710R Treadmill, PANLAB S.L.U.; Barcelona, Spain).

Procyanidins and Physical exercise group (GPEF) fed with fatty sugary cream and treated with GSPE (equal GP) and submitted to moderate physical exercise (equal GEF).

Each group was housed in independent cages equipped with feeders and free access to water and a special dietary food, provided ad libitum, and changed every day in a simultaneous way and at the same time $(9: 00 \mathrm{~h})$. The animals were submitted to a temperature of $22 \pm 1^{\circ} \mathrm{C}$ with $60 \% \pm 10 \%$ of relative humidity and a photoperiod of 12 hours of light/dark during all of the experiments in the animal house of the laboratory.

\section{Protocol of physical exercise}

The animals of the groups GEF and GPEF were acclimatized to the movement on a treadmill, to reduce the stress induced by the exercise, during the week before the period of experimentation (10 $\mathrm{min} /$ day at a slow march speed of $10 \mathrm{~m} / \mathrm{min}$; the first 3 days without inclination and last 3 days with $4^{\circ}, 6^{\circ}$ and $8^{\circ}$ of inclination respectively).

After this familiarization process, the 4-week physical exercise protocol (Table 1) began, in which both the velocity and the inclination were progressively increased. Before the beginning of every session, the animals performed a warm-up (5 min at a constant speed of $10 \mathrm{~m} /$ $\mathrm{min})$. Each week consisted of 6 sessions with duration of $60 \mathrm{~min}$ each, and a day of rest. The physical exercise sessions in the GEF and GPEF groups took place in the same time slot (12:00-14:00 h).

\begin{tabular}{|l|l|l|l|}
\hline Week & Speed $(\mathbf{m} / \mathbf{m i n})$ & Inclination & Time session $(\mathbf{m i n})$ \\
\hline 1 & 16 & $6^{\circ}$ & 60 \\
\hline 2 & 18 & $8^{\circ}$ & 60 \\
\hline 3 & 20 & $10^{\circ}$ & 60 \\
\hline 4 & 22 & $12^{\circ}$ & 60 \\
\hline
\end{tabular}

Table 1: Protocol of physical exercise moderated on rolling band.

The protocol carried out was of moderate intensity, since it is estimated that it ranges between $60-70 \%$ of the $\mathrm{VO}_{2}$ max for these types of animals. The proposed work intensities were based on the previous understanding of the metabolic responses of these types of animals to treadmill movement [13-18].

\section{Taking samples}

As soon as the experiment was finished, 8 hours after the last doses of procyanidins were given to the GP and GPEF groups, an individual examination of every animal was carried out to verify the absence of injuries. Each animal was then weighed and sedated by putting them in a sealed container, at the bottom of which was placed a swab soaked in ether, and later they were anesthetized with intra-peritoneal ketamine with a dose of $100 \mathrm{mg} / \mathrm{kg}$. After being sedated and anesthetized, blood samples were obtained from the abdominal aorta artery of each animal. The animals were finally euthanized by the administration of a lethal dose of sodium pentobarbital (120 mg/Kg intraperitoneal).

\section{Analysis of blood samples}

The plasmatic lipoproteins (CT, c-HDL, c-LDL, TG) and GL were measured using commercial colorimetric enzyme kits (QCA SA, Tarragona, Spain) following the instructions of the producer.

\section{Statistical analysis}

A descriptive statistical study was performed. The results were statistically verified using the Kruskal-Wallis test and the GamesHowell test for multiple comparisons. The comparison between the results obtained in the control group with the rest of the groups was 
Citation: Vega Vargas F, Guisado Requena IM, Canadas-De la Fuente GA, Castillo RF, Fernandez Sanchez E, et al. (2017) Effects of Combinational Procyanidins in Grape Seed Extract and Exercise on the Levels of Glucose and Blood Lipid Profile. J Diabetes Metab 8: 754. doi:10.4172/2155-6156.1000754

Page 3 of 5

done using the Dunnet test $t$. In all the cases work was carried out with a significance level of $\mathrm{p}<0.05$.

\section{Results}

The results relative to each of the dependent variables studied in each intervention or experimental group appear graphically in the Figure 1A-1E.
A

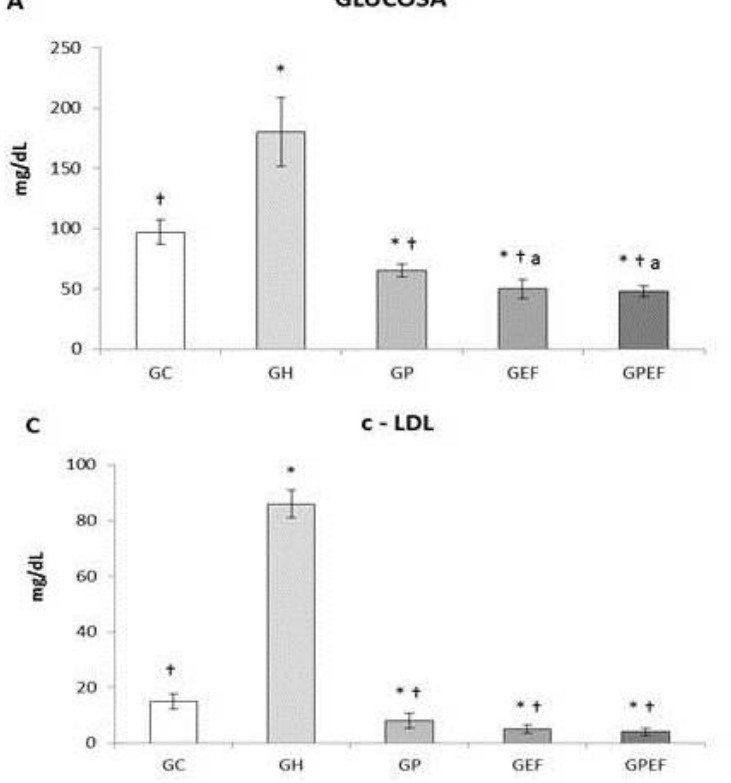

B

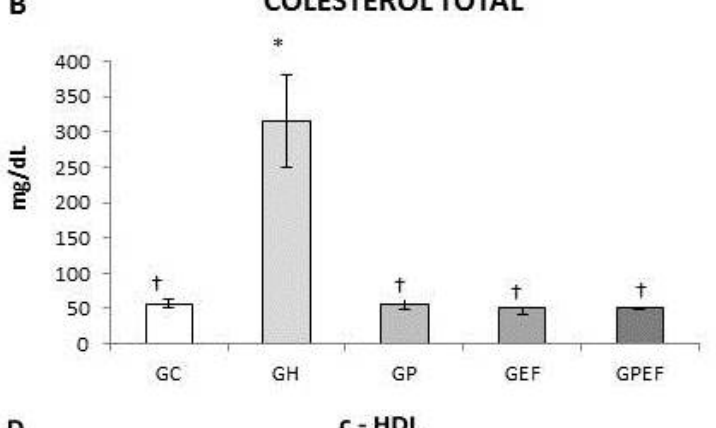

D

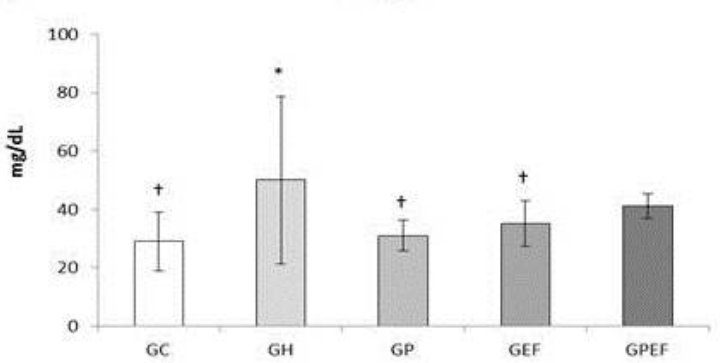

E

TRIGLICÉRIDOS

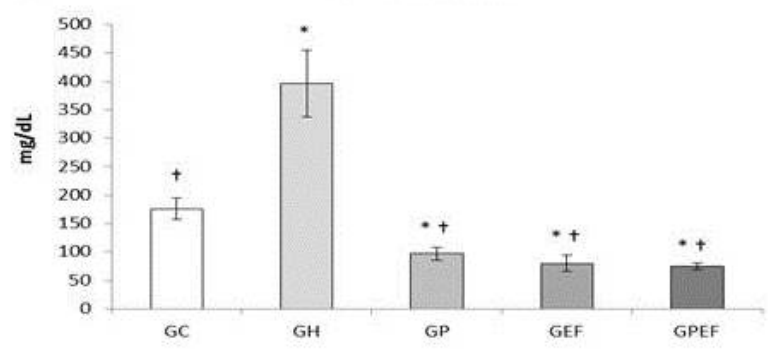

Figure 1: Results relative to each of the dependent variables studied in each intervention or experimental group.

\section{Glucose}

The glucose distribution is not the same between the different experimental groups $(\mathrm{p} \leq 0.001)$. The highest values were obtained in the GH group, with statistical significance when it was compared to the rest of the groups: GC ( $\mathrm{p} \leq 0.001), \mathrm{GP}(\mathrm{p}=0.004), \mathrm{GEF}(\mathrm{p} \leq 0.001)$ and GPEF ( $p=0.001$ ). In the GP, GEF and GPEF groups, the values of glycaemia are less with regard to the GC group with statistical significance ( $\mathrm{p}=0.004 ; \mathrm{p} \leq 0.001$; and $\mathrm{p}=0.001$ respectively). The lowest GL levels correspond to the GPEF group, with statistical significance when it is compared to the GP group ( $\mathrm{p}=0.007)$.

\section{Lipid sanguineous profile}

The distribution, of CT as well as c-LDL, c-HDL and TG, it is not the same between the different experimental groups $(\mathrm{p}=0.007 ; \mathrm{p} \leq$ $0.001 ; \mathrm{p}=0.004 ; \mathrm{p} \leq 0.001$ respectively). The highest values for CT, $\mathrm{c}-$ LDL, c-HDL, as in TG, were obtained in the GH group with statistical significance, when it was compared to the other groups.
In the GP, GEF and GPEF groups, CT values were lower and higher values of c-HDL compared to CG, although statistical significance was not obtained in any of them ( $p>0.05)$ except for the values of $c-H D L$ in GPEF that turned out to be significantly higher with regard to the GC $(\mathrm{p}=0.031)$. A decrease was observed in the LDL-C and TG levels, with statistical significance with regard to the GC in all three groups $(p=0.015 ; p \leq 0.001$ and $p \leq 0.001$ respectively for $c-L D L$, and $p=0.001$; $\mathrm{p} \leq 0.001$ and $\mathrm{p} \leq 0.001$ respectively for TG).

The percentage of c-HDL is clearly greater in the GC, GP, GEF and GPEF groups with regard to GH, with an inverse relation to c-LDL. Among the GP, GEF and GPEF groups, the biggest percentage of cHDL and a lower percentage of c-LDL were obtained in GPEF, with percentages of 77,73 and 82 respectively.

\section{Discussion}

The results obtained in this study show a significant reduction in the serum levels of GL, c-LDL and TG, in the GP, GEF and GPEF groups 
Citation: Vega Vargas F, Guisado Requena IM, Canadas-De la Fuente GA, Castillo RF, Fernandez Sanchez E, et al. (2017) Effects of Combinational Procyanidins in Grape Seed Extract and Exercise on the Levels of Glucose and Blood Lipid Profile. J Diabetes Metab 8: 754. doi:10.4172/2155-6156.1000754

Page 4 of 5

with regard to GC and GH, in addition to a reduction in the CT levels, although not significant. In addition, a significant increase in c-HDL in GPEF compared to the GP, which shows that, when administered together, both therapies resulted in a more significant improvement effect as well as a study of similar characteristics [19]. These results demonstrate a lipid lowering and hypoglycaemic effect of the treatment with GSPE, with physical exercise and both therapies have blood biochemical parameters in common.

Several intervention and biochemical studies have been done that demonstrate the potential for phenolic compounds to improve the different factors of risk associated with cardiovascular diseases. These compounds present vasodilator effects that are capable of improving the lipid profile and attenuate the LDL oxidation [5]. It has been demonstrated that the procyanidins possess a lipid lowering effect, at the same time that they provoke an increase in the levels of cholesterol tied to HDL, and a decrease of the levels of cholesterol tied to LDL [20-25]. It is probable that this effect is demonstrated across other mechanisms which are different to the reduction of lipids, like antioxidant activities, including the sweeping of hydroxyl and peroxyl radicals and the inhibition of the oxidation of LDL [26]. In the present study, an increase was obtained in the c-HDL/c-LDL quotient in plasma and a reduction in the values of TG in blood in the GP and GPEF groups, which represents a clearly anti-atherogenic situation.

Procyanidins also are formed like bioactive compounds that modulate the homeostasis of the GL [27]. Several studies have demonstrated a regulatory effect of procyanidins on the glycolytic homeostasis in animals' resistant to insulin [28,29]. In this study, a hypoglycaemic effect was observed, since in the GP and GPEF groups, a significant reduction was obtained in the blood GL levels with respect to the values obtained in the GC and GH groups, a fact that demonstrates that these compounds beneficially affect glycolytic homeostasis. A persistent increase in glycaemia results in the storage of glucose in the liver in the form of glycogen. This is emitted later into the circulatory system and through glycolysis is transformed into coenzyme A, capable of binding to proteins and thus gives rise to lipoproteins, which would explain the increase of c-LDL that is experienced in this situation [30-32].

The results of numerous studies [30-35], indicate that moderate physical exercise increases the plasma values of c-HDL and it reduces the plasma values of TG and c-LDL. Nevertheless, the type, intensity, frequency, and the ideal duration of the physical exercise, are variables that are not yet widely agreed upon. In this study, after a treatment of 4 weeks with moderate regular physical exercise, of aerobic type (GEF) and combined with a treatment with procyanidins (GPEF), a reduction was obtained in the levels of TG (with statistical significance) and of $\mathrm{CT}$, in each group, with respect to the values obtained in the GC and GH groups. Therefore, according to the results obtained, the practice of moderate physical exercise might be associated with better functionality of the cardiovascular system because of the improvements that it produces in the lipid blood profile.

It has been demonstrated that the training programs for people with have beneficial effects on weight loss, fasting glucose levels, fasting insulin levels, as well as the resistance to insulin, valuation of lipid oxidation, cholesterol levels in blood and blood pressure [36]. The results that were obtained in the present study show a reduction in the GL levels in blood, both in the GEF and in GPEF groups. This hypoglycaemic effect, mediated by the practice of moderate physical exercise, has resulted in significant levels in relation to the CG group and, more markedly, in relation to the $\mathrm{GH}$ group.

\section{Conclusions}

Procyanidins from grape seed extract and moderate physical exercise, show a lipid lowering and glucose lowering effect, both in isolation and in combination, in treated versus non-treated animals. Therefore, based on the results obtained in this study and considering the consulted bibliographical precedents, these substances and physical exercise are postulated as being very useful potential tools to preserve the balance, the natural conditions and the functions of the vascular endothelium.

\section{Acknowledgements}

We would like to express our gratitude to the Departments of Nursing and of Surgery and its Specialties of the University of Granada for the facilities they have given us in the realization of this study.

\section{References}

1. Miller LW, Missov ED (2001) Epidemiology of heart failure. Cardiol Clin 19: 547-55.

2. Nishizuka T, Fujita Y, Sato Y, Nakano A, Kakino A, et al. (2011) Procyanidins are potent inhibitors of LOX-1: a new player in the French Paradox. Proc Jpn Acad 87: 104-13.

3. Del Bas JM, Ricketts ML, Baiges I, Quesada H, Ardevol A, et al. (2008) Dietary procyanidins lower triglyceride levels signaling through the nuclear receptor small heterodimer partner. Mol Nutr Food Res 52: 1172-1181.

4. Bladé C, Arola L, Salvadó MJ (2010) Hypolipidemic effects of proanthocyanidins and their underlying biochemical and molecular mechanisms. Mol Nutr Food Res 54: 37-59.

5. Quiñones M, Miguel M, Aleixandre A (2012) Los polifenoles, compuestos de origen natural con efectos saludables sobre el sistema cardiovascular. Nutr Hosp 27: 76-89.

6. Pagels P, Raustorp A, Archer T, Lidman U, Alricsson M (2012) Influence of moderate, daily physical activity on body composition and blood lipid profile in Swedish adults. J Phys Act Health 9: 867-874.

7. Barzegari A, Amouzad Mahdirejei H (2014) Effects of 8 weeks resistance training on plasma vaspin and lipid profile levels in adult men with type 2 diabetes. Caspian J Intern Med 5: 103-108.

8. Speretta GF, Rosante MC, Duarte FO, Leite RD, Lino AD, et al. (2012) The effects of exercise modalities on adiposity in obese rats. Clinics (Sao Paulo) 67: 1469-1477.

9. Pérez-Terzic CM (2012) Exercise in cardiovascular diseases. PMR 4 867-873.

10. Thijssen DHJ, Maiorana AJ, O'Driscoll G, Cable NT, Hopman MTE, et al. (2010) Impact of inactivity and exercise on the vasculature in humans. Eur J Appl Physiol 108: 845-875.

11. Belviranli M, Gökbel H, Okudan N, Büyükbas S (2013) Effects of grape seed polyphenols on oxidative damage in liver tissue of acutely and chronically exercised rats. Phytother Res 27: 672-677.

12. Bedford TG, Tipton CM, Wilson NC, Oppliger RA, Gisolfi CV (1979) Maximum oxygen consumption of rats and its changes with various experimental procedures. J Appl Physiol Respir Environ Exerc Physiol 47: 1278-1283.

13. Billat VL, Mouisel E, Roblot N, Melki J (2005) Inter- and intrastrain variation in mouse critical running speed. J Appl Physiol 98: 1258-1263.

14. Bupha-Intr T, Wattanapermpool J (2004) Cardioprotective effects of exercise training on myofilament calcium activation in ovariectomized rats. J Appl Physiol 96: 1755-1760.

15. Cechetti F, Worm PV, Elsner VR, Bertoldi K, Sanches E, et al. (2012) Forced treadmill exercise prevents oxidative stress and memory deficits following chronic cerebral hypoperfusion in the rat. Neurobiol Learn Mem 97: 90-96. 
Citation: Vega Vargas F, Guisado Requena IM, Canadas-De la Fuente GA, Castillo RF, Fernandez Sanchez E, et al. (2017) Effects of Combinational Procyanidins in Grape Seed Extract and Exercise on the Levels of Glucose and Blood Lipid Profile. J Diabetes Metab 8: 754. doi:10.4172/2155-6156.1000754

Page 5 of 5

16. Miller LE, Hosick PA, Wrieden J, Hoyt E, Quindry JC (2012) Evaluation of arrhythmia scoring systems and exercise-induced cardioprotection. Med Sci Sports Exerc 44: 435-41.

17. Wen S, Jadhav KS, Williamson DL, Rideout TC (2013) Treadmill Exercise Training Modulates Hepatic Cholesterol Metabolism and Circulating PCSK9 Concentration in High-Fat-Fed Mice. J Lipids 2013: 908048.

18. Ito D, Ito O, Cao P, Mori N, Suda C, et al. (2013) Effects of exercise training on nitric oxide synthase in the kidney of spontaneously hypertensive rats. Clin Exp Pharmacol Physiol 40: 74-82.

19. Badavi M, Abedi HA, Dianat M, Sarkaki AR (2013) Exercise Training and Grape Seed Extract Co-Administration Improves Lipid Profile, Weight Loss, Bradycardia, and Hypotension of STZ-Induced Diabetic Rats. Int Cardiovasc Res J 7: 111-117.

20. Preuss HG, Wallerstedt D, Talpur N, Tutuncuoglu SO, Echard B, et al. (2000) Effects of niacin-bound chromium and grape seed proanthocyanidin extract on the lipid profile of hypercholesterolemic subjects: a pilot study. J Med 31: 227-246.

21. Vinson JA, Mandarano MA, Shuta DL, Bagchi M, Bagchi D (2002) Beneficial effects of a novel IH636 grape seed proanthocyanidin extract and a niacin-bound chromium in a hamster atherosclerosis model. $\mathrm{Mol}$ Cell Biochem. 240: 99-103.

22. Auger C, Gérain P, Laurent-Bichon F, Portet K, Bornet A, et al. (2004) Phenolics from commercialized grape extracts prevent early atherosclerotic lesions in hamsters by mechanisms other than antioxidant effect. J Agric Food Chem 52: 5297-302.

23. Thiruchenduran M, Vijayan NA, Sawaminathan JK, Devaraj SN (2011) Protective effect of grape seed proanthocyanidins against cholesterol cholic acid diet-induced hypercholesterolemia in rats. Cardiovasc Pathol 20: 361-368.

24. Caimari A, del Bas JM, Crescenti A, Arola L (2013) Low doses of grape seed procyanidins reduce adiposity and improve the plasma lipid profile in hamsters. Int J Obes (Lond) 37: 576-583.

25. Del Bas JM, Fernández-Larrea J, Blay M, Ardèvol A, Salvadó MJ, et al. (2005) Grape seed procyanidins improve atherosclerotic risk index and induce liver CYP7A1 and SHP expression in healthy rats. FASEB J 19 : 479-481.
26. Feringa HH, Laskey DA, Dickson JE, Coleman CI (2011) The effect of grape seed extract on cardiovascular risk markers: a meta-analysis of randomized controlled trials. J Am Diet Assoc 111: 1173-1781.

27. Pinent M, Cedó L, Montagut G, Blay M, Ardévol A (2012) Procyanidins improve some disrupted glucose homoeostatic situations: an analysis of doses and treatments according to different animal models. Crit Rev Food Sci Nutr 52: 569-84.

28. Montagut G, Blade C, Blay M, Fernández-Larrea J, Pujadas G, et al. (2009) Effects of a grape seed procyanidin extract (GSPE) on insulin resistance. J Nutr Biochem 21: 961-967.

29. Pinent M, Blay M, Bladé MC, Salvadó MJ, Arola L, et al. (2004) Grape seed-derived procyanidins have an antihyperglycemic effect in streptozotocin-induced diabetic rats and insulinomimetic activity in insulin-sensitive cell lines. Endocrinology 145: 4985-4990.

30. Fahlman MM, Boardley D, Lambert CP, Flynn MG (2002) Effects of endurance training and resistance training on plasma lipoprotein profiles in elderly women. J Gerontol A Biol Sci Med Sci 57: B54-B60.

31. Kelley GA, Kelley KS (2009) Impact of progressive resistance training on lipids and lipoproteins in adults: a meta-analysis of randomized controlled trials. Prev Med 48: 9-19.

32. Greene NP, Martin SE, Crouse SF (2012) Acute exercise and training alter blood lipid and lipoprotein profiles differently in overweight and obese men and women. Obesity (Silver Spring). 20: 1618-1627.

33. Durstine JL, Grandjean PW, Cox CA, Thompson PD (2002) Lipids, lipoproteins, and exercise. J Cardiopulm Rehabil 22: 385-398.

34. Durstine JL, Grandjean PW, Davis PG, Ferguson MA, Alderson NL, et al. (2001) Blood lipid and lipoprotein adaptations to exercise: a quantitative analysis. Sports Med 31: 1033-1062.

35. Halbert JA, Silagy CA, Finucane P, Withers RT, Hamdorf PA (1999) Exercise training and blood lipids in hyperlipidemic and normolipidemic adults: a meta-analysis of randomized, controlled trials. Eur J Clin Nutr 53: 514-522.

36. O'Hagan C, De Vito G, Boreham CA (2013) Exercise prescription in the treatment of typediabetes mellitus: current practices, existing guidelines and future directions. Sports Med 43: 39-49. 